\title{
ROS Promote Ox-LDL-Induced Platelet Activation by Up-Regulating Autophagy Through the Inhibition of the PI3K/AKT/ mTOR Pathway
}

\author{
Xiang Wang Yun-Feng Fu Xiao Liu Guo Feng Dan Xiong Guang-Fu Mu \\ Fang-Ping Chen
}

The Third Xiangya Hospital of Central South University, Changsha, P.R. China

\section{Key Words}

Ros $•$ PI3K/AKT/mTOR signaling pathway • Autophagy $•$ Ox-LDL $・$ Platelet activation

\begin{abstract}
Background/Aims: Oxidized low-density lipoprotein (OxLDL) promotes unregulated platelet activation in patients with dyslipidemic disorders. Although oxLDL stimulates activating signaling, researchers have not clearly determined how these events drive accelerated thrombosis. Here, we describe the mechanism by which ROS regulate autophagy during ox-LDL-induced platelet activation by modulating the PI3K/AKT/mTOR signaling pathway. Methods: For in vitro experiments, ox-LDL, the ROS scavenger $\mathrm{N}$-acetylcysteine (NAC), the mTOR inhibitor rapamycin and the autophagy inhibitor 3-MA were used alone or in combination with other compounds to treat platelets. Then, platelet aggregation was evaluated on an aggregometer and platelet adhesion was measured under shear stress. The levels of a platelet activation marker (CD62p) were measured by flow cytometry, reactive oxygen species (ROS) levels were then quantified by measuring DCFH-DA fluorescence intensity via flow cytometry. Nitric oxide (NO) and superoxide $\left(\mathrm{O}_{2}^{-}\right)$levels were determined by the nitric acid deoxidize enzyme method and lucigenin-enhanced chemiluminescence $(\mathrm{CL})$, respectively. Transmission electron microscopy was used to observe the autophagosome formation, immunofluorescence staining was employed to detect LC3 expression and western blotting was used to measure the levels of PI3K/AKT/mTOR pathway- and autophagy-related proteins. Results: Ox-LDLinduced platelets showed a significant increase in platelet aggregation and adhesion, CD62p expression, $\mathrm{ROS}$ level and $\mathrm{O}_{2}{ }^{-}$content, with an elevated $\mathrm{LC} 3 \mathrm{II} / \mathrm{LC} 3 \mathrm{I}$ ratio and Beclin 1 expression, but a dramatic reduction in the levels of p62 and pathway-related proteins (all $P<0.05$ ). However, platelet activation and autophagy were aggravated by the Rapamycin treatment, and decreased following treatment with NAC, 3-MA, or NAC and 3-MA, together with increased activity of the PI3K/AKT/mTOR pathway. Additionally, decreased platelet activation and autophagy were observed in platelets treated with NAC and Rapamycin or Rapamycin
\end{abstract}

\begin{tabular}{ll}
\hline Fang-Ping Chen & The Third Xiangya Hospital of Central South University \\
& Changsha 410013 (China) \\
& Tel. +86-0731-88638888, E-Mail med-rong@outlook.com
\end{tabular}


and 3-MA compared with platelets treated with Rapamycin alone, suggesting that both NAC and 3-MA reversed the effects of Rapamycin. Conclusion: Inhibition of ROS production may reduce autophagy to suppress ox-LDL-induced platelet activation by activating PI3K/AKT/ mTOR pathway.

(C) 2018 The Author(s)

Published by S. Karger AG, Basel

\section{Introduction}

Atherosclerosis (AS), a leading cause of death in developed countries, may result in myocardial ischemia, myocardial infarction, stoke and other cardio-/cerebrovascular diseases, seriously affecting human health and patients' quality of life $[1,2]$. AS is a complex disease associated with various genetic and environmental factors, and is mainly triggered by oxidative stress and the inflammatory response [3]. Reactive oxygen species (ROS) generated by oxidative stress, which are well-known important initiating factors required for AS development, oxidize low-density lipoprotein (LDL) to form oxidized-LDL (ox-LDL) $[4,5]$. Moreover, ox-LDL, a main factor contributing to endothelial dysfunction, induces the autophagy of vascular endothelial cells, thereby leading to AS, as reported by several researchers [6, 7]. For example, according to Peng $\mathrm{N}$ et al., ox-LDL inhibits mammalian target of rapamycin (mTOR) and induces autophagy and apoptosis in vascular endothelial cells (VECs), which play very critical roles in cardiovascular homeostasis [8]. More importantly, ox-LDL has been implicated in the early phase of atherosclerosis by recruiting inflammatory cells in the sub-endothelium and eliciting changes in endothelial function that favor the thrombotic process [7]. Ox-LDL also stimulates platelet activation to induce the expression of pro-inflammatory cytokines, such as adhesion molecules and thrombogenic tissue factor, which play crucial roles in pro-atherogenic mechanisms [9-11]. Thus, the protection of endothelial cells against ox-LDL-induced platelet activation and injury is a very important strategy to prevent and treat AS-related diseases.

Autophagy, a highly conserved pathway mediated by lysosomes that degrades cytosolic components in eukaryotic cells [12], is essential for maintaining cell survival and protein homeostasis during normal development, and its dysfunction is involved in many human diseases $[13,14]$. Recently, the role of autophagy in platelets has gradually been recognized; for example, some unidentified forms of platelet dysfunction might be caused by abnormal autophagy, resulting in severe hemostasis and thrombosis defects, as illustrated by Ouseph et al. [15]. Additionally, Feng et al. also reported the induction of autophagy in platelets by cell starvation or rapamycin (mTOR inhibitor) treatment [16]. However, the specific mechanism of autophagy in platelets has not yet been elucidated. On the other hand, the phosphoinositide 3-kinase (PI3K)/protein kinase B (AKT)/mammalian target of rapamycin (mTOR) pathway, a classic autophagy pathway [17], is well established as a regulator of a wide range of cellular processes that mediate cell survival and death, and this pathway is inhibited by malnutrition, hypoxia, external pressure or other stress conditions to suppress cell proliferation and enhance cell autophagy, eventually leading to cell death $[16,18]$. Moreover, suppression of PI3K and Akt signaling inhibits mTOR phosphorylation at Ser2448, thereby inducing autophagy and increasing the levels of autophagy-related proteins $[18,19]$. Recently, ROS was shown to play a regulatory role in autophagy in various cells by modulating the PI3K/AKT/mTOR pathway. For instance, in the study by Jiang et al., hydroxysafor yellowmediated sonodynamic therapy not only repressed the PI3K/AKT/mTOR signaling pathway to trigger an autophagy response but also suppressed ROS-induced inflammation in THP1 macrophages [20]. Furthermore, the important influence of the ROS/PI3K/AKT/mTOR signaling pathway was also highlighted by Han et al. in photodynamic therapy-induced autophagy in THP-1 and peritoneal macrophage-derived foam cells [21]. Therefore, we aim to explore whether ROS regulate platelet autophagy by modulating PI3K/AKT/mTOR signaling pathway. Notably, N-acetylcysteine (NAC), a well-known ROS scavenger that is widely utilized to identify and test ROS inducers and to decrease the intracellular ROS levels, has shown therapeutic potential as an antiplatelet agent due to its inhibitory effect on platelet 
hyperaggregability in patients with type-2 diabetes [22]. Meanwhile, 3-methyladenine (3MA), a classic autophagy inhibitor, blocks autophagosome formation by inhibiting type III phosphatidylinositol 3-kinases in platelets [23], and the specific mTOR inhibitor, rapamycin, the most frequently used trigger of autophagy, has been used to inhibit mammalian target of rapamycin (mTOR) function in platelets [24]. Thus, in this study, ox-LDL, NAC, Rapamycin, or 3-MA was administered alone or in combination with other compounds to platelets to improve our understanding of the potential molecular effects of these compounds on ROSinduced autophagy and platelet activation.

\section{Materials and Methods}

\section{Ethics statement}

All subjects provided written informed consent to participate in this study, which was approved by the Ethics Committee of Clinical Laboratory in our hospital. All procedures performed in this study adhered to the guidelines of the Declaration of Helsinki [25].

\section{Preparation of ox-LDL and washed platelets}

Whole blood was collected from healthy human volunteers at The Third Xiangya Hospital of Central South University. We prepared ox-LDL using a previously reported method [26]. The blood was mixed with $0.109 \mathrm{M}$ sodium citrate in 1:9 ratio as an anti-freeze. One hundred fifty grams of the mixture were centrifuged for $20 \mathrm{~min}$ to obtain platelet-rich plasma (PRP). We only removed the upper two-thirds of the PRP fraction to prevent contamination with other cell types. Based on the results from a previous study, the gel was filtered using a Sepharose 4B column in HEPES-Tyrode's buffer supplemented with $0.5 \%$ human serum albumin [3]. Then, the gel or PGI2 $(1 \mu \mathrm{M}$; Sigma-Aldrich, USA) was used to centrifuge $(3000 \times \mathrm{g}, 2$ min) platelets.

\section{Grouping and treatment of washed platelets}

The washed platelets were divided into 8 groups in equal volumes: the Control group (platelets treated with the vehicle, dimethyl sulfoxide (DMSO) (Sigma-Aldrich, USA)); ox-LDL group (platelet suspension treated with $50 \mu \mathrm{g} / \mathrm{ml}$ ox-LDL for $5 \mathrm{~min}$ ) [27]; N-acetylcysteine (NAC) group; Rapamycin group; 3-methyladenine (3-MA) group; NAC + Rapamycin group; NAC + 3-MA group; and Rapamycin + 3-MA group. The suspended platelets in each group were pre-treated with a 1-mM dose of the ROS scavenger N-acetylcysteine (NAC) (Sigma-Aldrich, USA) [28], a 0.2- $\mu \mathrm{M}$ dose of the mTOR inhibitor Rapamycin (Sigma-Aldrich, USA) [16] or a 1-mM dose of the autophagy inhibitor 3-MA (Sigma-Aldrich, USA) [16] for $30 \mathrm{~min}$, and then $50 \mu \mathrm{g} / \mathrm{ml} \mathrm{ox}$ LDL was added to platelets and incubated for $10 \mathrm{~min}$.

\section{Scanning electron microscopy}

Platelets $\left(4 \times 10^{7} / \mathrm{ml}\right)$ were fixed with $4 \%$ paraformaldehyde to examine the platelet morphology in solution. Subsequent procedures (including dehydration and critical-point drying) were performed using standard methods, and the coated specimens were examined under a JEOL 6400 scanning electron microscope (JEOL, Tokyo, Japan) with automated image digitization and archiving.

\section{Determination of platelet aggregation}

A total of $4 \mu \mathrm{M}$ adenosine diphosphate (ADP) was added to trigger platelet aggregation. Then, an aggregometer (Lumi-Aggregometer, Canada) was used to measure changes in turbidity to determine the degree of platelet aggregation at $37^{\circ} \mathrm{C}$ with stirring at $900 \mathrm{rpm}$. The quantification of aggregation is presented as the ratio of the treated group to the control.

\section{Platelet adhesion under shear stress}

After platelets were diluted with PBS at a 1:10 ratio, platelets were perfused over collagen IV-coated channel slides (iBiDi, Martinsried, Germany) for $2 \mathrm{~min}$ and monitored by capturing real-time phase contrast images using a microscopy. We set the flow rate to $5 \mathrm{ml} / \mathrm{min}$ to obtain a shear rate of approximately 1000 $\mathrm{s}^{-1}$. Next, slides were washed with PBS for $5 \mathrm{~min}$ and 5 isolated fields along the centerline of the channel 


\section{Cellular Physiology Cell Physiol Biochem 2018;50:1779-1793 and Biochemistry Published \begin{tabular}{l|l}
$\overline{\text { DOI } 10.1159 / 000494795}$ & $\begin{array}{l}\text { (c) } 2018 \text { The Author(s). Published by S. Karger AG, Basel } \\
\text { www.karger.com/cpb }\end{array}$
\end{tabular}}

Wang et al.: ROS in Ox-LDL-Induced Platelet Activation

were observed under the microscope and analyzed using ImagePro Software (Version 5.0, United Kingdom, 2003) and Image (Version 1.45s, USA, 2012). Platelet adhesion was calculated as the ratio of the treated group to collagen (fold changes).

\section{Flow cytometry}

Platelets were incubated and detected using flow cytometry. Two Falco sample tubes were labeled A and B: A was the control tube for the same type and B was the determination tube. Then, $20 \mu \mathrm{l}$ of CD61 Per $\mathrm{CP}$ antibody (BD Biosciences, USA) were separately added to tubes A and B. We added $20 \mu \mathrm{l}$ of the same species of Ig G control antibody (BD Biosciences, USA) to tube A and $20 \mu \mathrm{l}$ of CD62p PE (BD Biosciences, USA) to tube B. Next, $5 \mu \mathrm{l}$ of platelets and $200 \mu \mathrm{l}$ of Tyrode's buffer were added to each tube and mixed with the aforementioned liquids in the bottom of the tube. After a 20 min incubation at room temperature in the dark, a FACSCalibur flow cytometer (BD Biosciences, USA) equipped with Cell Quest software was used to collect and analyze the data, and platelets labeled with CD61 were used to determine the percentage of CD62p-positive platelets.

\section{Detection of ROS production}

Intracellular ROS measurements were performed by measuring the fluorescence intensity of 2 , 7-dichlorofluorescein (DCF). The platelets in each group was incubated with the fluorescent probe 2, 7-dichlorodihydrofluorescindiacetate (DCFH-DA) $(1: 1000)$ at $37^{\circ} \mathrm{C}$ for $30 \mathrm{~min}$ in the dark, and then washed three times. The fluorescence signals produced were analyzed using a FACSVese flow cytometer (BD, Germany). Fluorescence was measured with a fluorescence microscope (Olympus IX81, Japan) at $488 \mathrm{~nm}$ (excitation) and $525 \mathrm{~nm}$ (emission) wavelengths, and photos were captured.

\section{Determination of the Nitric oxide (NO) content and superoxide anion $\left(\mathrm{O}_{2}^{-}\right)$level in platelets}

The NO detection kit (nitric acid deoxidize enzyme method) was provided by Nanjing Jiancheng Bioengineering Institutes. Platelets were centrifuged at $3500 \mathrm{~g}$ for $15 \mathrm{~min}$, and aliquots of $100 \mu \mathrm{l}$ of supernatant were mixed with an equal volume of Griess reagent (containing $1 \%$ sulphanilamide and $0.1 \%$ naphthlethylenediamine in 5\% phosphoric acid). Platelets were first incubated at room temperature for 10 min and then their absorbance $(550 \mathrm{~nm})$ was measured using a micro-plate reader (Titertek multiskan MCC). Solutions of sodium nitrite were used as standards. The nitrite concentrations in platelets were calculated by comparing their OD values with standard solutions. The results are presented as NO release corrected for platelet counts in picomoles per $10^{8}$ platelets. The specific procedures were performed according to the manufacturer's instructions. Lucigenin-enhanced chemiluminescence (CL) was used to determine the platelet $02-$ level. Platelets were first equilibrated in Krebs solution at $37^{\circ} \mathrm{C}$ and then equilibrated in KrebsHEPES buffer at $37^{\circ} \mathrm{C}$ for $30 \mathrm{~min}$. Platelets were then placed in $0.5 \mathrm{ml}$ of Krebs-HEPES buffer containing 250 $\mathrm{mmol} / \mathrm{L}$ lucigenin at $37^{\circ} \mathrm{C}$ in a Picolite luminometer (Packard), and luminescence counts were measured every minute for $15 \mathrm{~min}$. Background counts were determined for 15 min before adding the platelets; these values were then subtracted from the luminescence counts, and the results are presented as $\mathrm{O}_{2}$. concentrations in picomoles per $10^{8}$ platelets.

\section{Observation of autophagosome formation by transmission electron microscopy}

The suspended platelets in each group were pelleted twice for fixation with a $2.5 \%$ glutaraldehyde solution and a $1 \%$ osmium tetroxide solution. Finally, the platelets were dehydrated with an ethanol solution, embedded and cut into ultrathin sections with a thickness of $100 \mathrm{~nm}$. After staining with a saturated uranyl acetate solution and a lead acetate solution, the platelets were observed and photographed under a transmission electron microscope.

\section{Detection of LC3 expression by immunofluorescence staining}

After grouping, platelets were fixed with $4 \%$ paraformaldehyde for $20 \mathrm{~min}$, washed three times and sealed with sealing solution. After the sealing solution was removed, the platelets were incubated with a primary antibody against LC3 (1:50) at $4^{\circ} \mathrm{C}$ overnight, followed by washes with PBS. Next, a fluorescent phycoerythrin (PE)-labeled secondary antibody was added to platelets and incubated for $1 \mathrm{~h}$ at room temperature in the dark; platelets were then washed with PBS for 3 times. After adding 40-6-diamidino- 


\section{Cellular Physiology Cell Physiol Biochem 2018;50:1779-1793

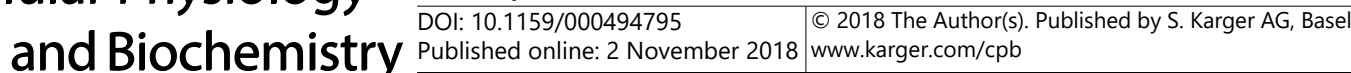

Wang et al.: ROS in Ox-LDL-Induced Platelet Activation

2-phenylindole (DAPI) and incubating the platelets with the dye at room temperature for 10 min, platelets were sealed with anti-fluorescence quenching liquid, observed and photographed under a fluorescence microscope.

\section{Western blotting}

According to instructions of the BCA (bicinchoninic acid) protein assay (Beyotime Biotechnology Co., Shanghai, China), cell proteins were extracted to determine the protein concentration. Loading buffer was added to the extracted proteins and boiled at $95^{\circ} \mathrm{C}$ for $10 \mathrm{~min}$. Thirty micrograms of protein sample were loaded into each well of a 10\% polyacrylamide gel (Wuhan Boster Biological Technology Ltd., Wuhan, Hubei, China) and separated by electrophoresis. Then, the proteins were transferred to a polyvinylidene fluoride (PVDF) membrane and incubated with $5 \%$ bovine serum albumin (BSA) at room temperature for $1 \mathrm{~h}$. Next, the PVDF membrane was incubated with primary antibodies against, p-PI3K, PI3K, phosphorylated (p)AKT, AKT, p-mTOR, mTOR, LC3, Beclin1, p62 and GADPH (Cell Signaling Technology, Inc.) at 1:1000 dilutions overnight at $4^{\circ} \mathrm{C}$. After three washes with Tris-buffered saline containing Tween (TBST) for 5 min each, the signals on the PVDF membrane were developed by incubating the membrane with chemiluminescence reagents; GADPH was used as the internal reference. The gray value of each target band was analyzed using ImageJ software.

\section{Statistical analysis}

All data were analyzed with SPSS 21.0 software (SPSS Inc., Chicago, IL, USA), and values are reported as means \pm standard deviation $(\bar{x} \pm s$ ). All experiments were repeated three times to obtain the mean value and standard deviation. The data from multiple groups were compared using one-way ANOVA, whereas pairwise comparisons between two groups were performed using t-tests. A $P$ value of $<0.05$ was considered statistically significant.

\section{Results}

Platelet aggregation and adhesion in each group

Scanning electron micrographs revealed that ox-LDL induced the formation of platelet microaggregates (Fig. 1A). Platelets in the Rapamycin group formed large, stable aggregates. However, NAC, 3-MA and NAC + 3-MA clearly prevented aggregate formation. Compared with the Control group, the ox-LDL and Rapamycin groups exhibited an evident increase in platelet aggregation and adhesion (all $P<0.05$ ). Moreover, platelet aggregation and adhesion were substantially increased in the Rapamycin group (both $P<0.05$ ), but were dramatically decreased in the NAC, 3-MA and NAC + 3-MA groups compared to the ox-LDL group (all $P<$ 0.05). Significant differences in platelet aggregation and adhesion were not observed among the Rapamycin + 3-MA, NAC + Rapamycin and ox-LDL groups (all $P>0.05$ ). Meanwhile, the Rapamycin + 3-MA and NAC + Rapamycin groups showed significant reductions in platelet aggregation and adhesion compared with the Rapamycin group (all $P<0.05$, Fig. $1 \mathrm{~B}$ and $\mathrm{C}$ ). Based on these findings, ox-LDL-treated platelets aggregated, whereas the ROS scavenger NAC and the autophagy inhibitor 3-MA reversed ox-LDL-induced platelet aggregation.

\section{Expression of platelet activation markers in each group}

As shown in Fig. 2, a greater percentage of platelets in the ox-LDL group and rapamycin group expressed CD62p than the Control group and ox-LDL group, respectively (both $P<$ 0.05). In contrast, a critical decrease in the percentage of platelets expressing CD62p was observed in the NAC group, 3-MA group and NAC + 3-MA group (all $P<0.05$ ). No significant differences were observed among the ox-LDL, Rapamycin + 3-MA, and NAC + Rapamycin groups (all $P>0.05$ ). However, the percentages of CD62p-positive platelets were noticeably decreased in the Rapamycin + 3-MA group and NAC + Rapamycin group compared with the Rapamycin group (both $P<0.05$ ). 
Fig. 1. Comparison of platelet aggregation and adhesion in each group. Notes: A, Scanning electron micrographs reveal that ox-LDL induces the formation of platelet microaggregates (scale bar: 1 $\mu \mathrm{m})$. B, Platelet aggregation was monitoredina Lumi-Aggregometer, and the quantification of platelet aggregation is shown. C, Quantification of platelet adhesion to a collagen-coated surface under high shear rates $\left(1000 \mathrm{~s}^{-1}\right)$. All data are presented as means \pm standard deviations. Different letters refer to significant differences, while the same letters do not indicate significant differences among groups.
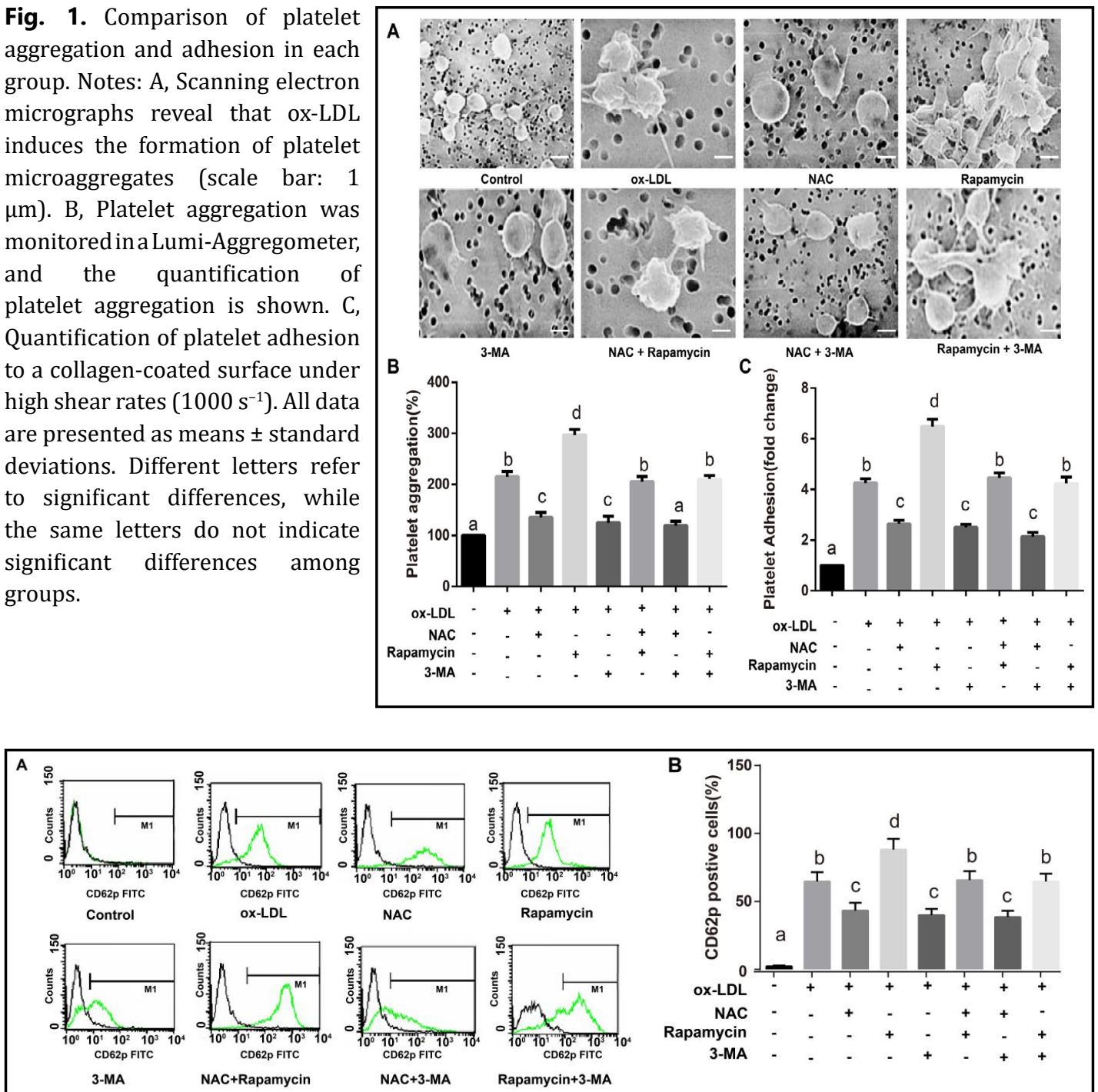

Fig. 2. Comparison of the expression of a platelet activation marker (CD62p) in each group. Notes: A, Platelets in the ox-LDL, NAC, Rapamycin, 3-MA, NAC + Rapamycin, NAC + 3-MA and Rapamycin + 3-MA groups were stimulated with $50 \mu \mathrm{g} / \mathrm{ml}$ ox-LDL; platelets were collected, stained with CD62p monoclonal antibodies, and subjected to flow cytometry. B, Histogram showing the percentages of CD62p-positive platelets in each group. All data are presented as means \pm standard deviations. Different letters refer to significant differences, while the same letters do not indicate significant differences among groups.

\section{Comparison of ROS contents in each group of platelets}

Fluorescence microscopy was used to observe the fluorescence intensity in each group. Compared with Control group, the fluorescence intensity was increased and the ROS level was substantially increased in the ox-LDL group (both $P<0.05$ ). Compared to the ox-LDL group, the ROS level was significantly increased in the Rapamycin group $(P<0.05)$, but was decreased in the NAC group, 3-MA group and NAC + 3-MA group (all $P<0.05$ ). Both the Rapamycin + 3-MA group and NAC + Rapamycin group exhibited a noticeable reduction in the ROS level compared with the Rapamycin group $(P<0.05)$. No significant changes were observed between the rapamycin + 3-MA, NAC + Rapamycin, and ox-LDL groups (all $P>0.05$, Fig. 3). 


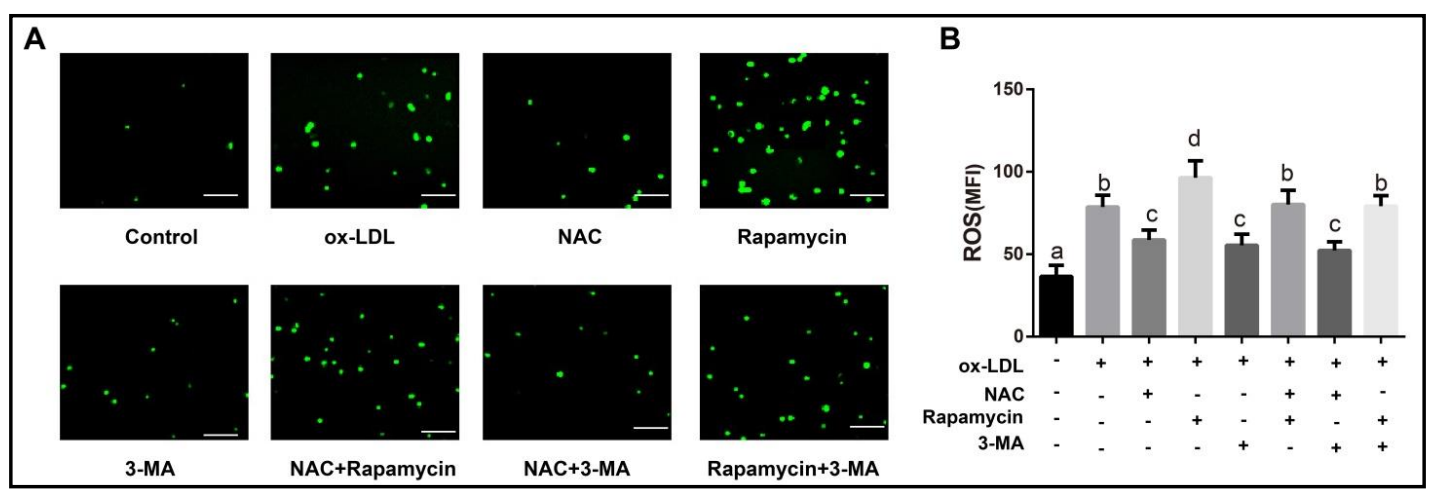

Fig. 3. Intracellular ROS generation in platelets was measured using DCFH-DA staining. Notes: A, Representative fluorescence microscopy images of ROS staining (scale bar: $50 \mu \mathrm{m}$ ). Platelets in the ox-LDL, NAC, Rapamycin, 3-MA, NAC + Rapamycin, NAC + 3-MA and Rapamycin + 3-MA groups were stimulated with $50 \mu \mathrm{g} / \mathrm{ml}$ ox-LDL. B, Comparison of ROS contents in platelets from each group. All data are presented as means \pm standard deviations. Different letters refer to significant differences, while the same letters do not indicate significant differences among groups.

Fig.

Comparison of platelet nitric oxide (NO) and superoxide $\left(\mathrm{O}_{2}{ }^{-*}\right)$ release from each group. Notes: A, Histogram $\mathrm{s}$ h $\mathrm{o}$ w $\mathrm{i}$ n $\mathrm{g}$ endogenous NO production in platelets treated with the different

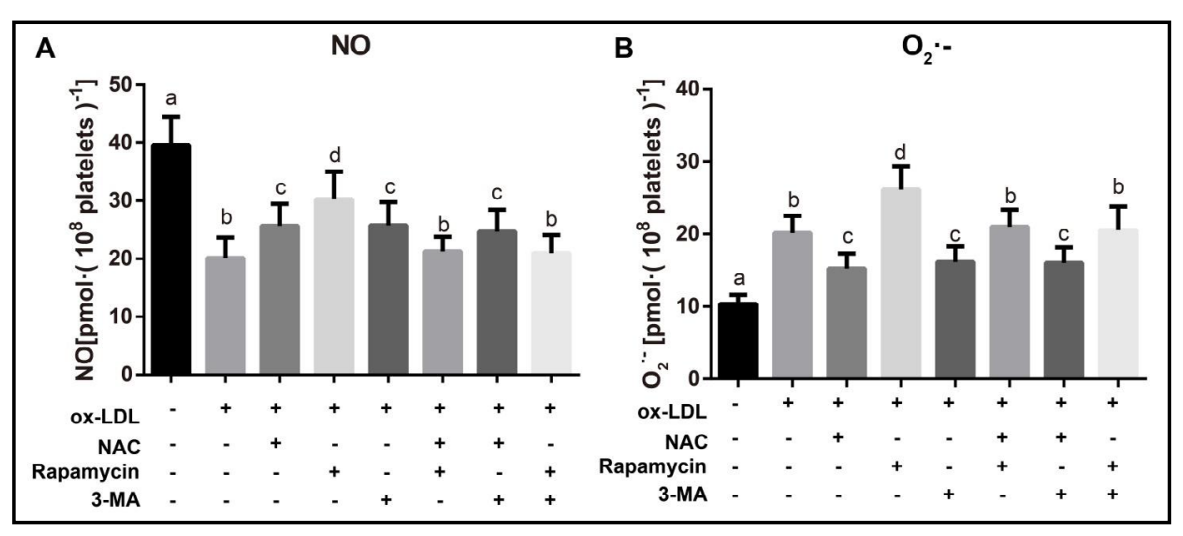

compounds. B, Histograms show the quantification of $\mathrm{O}_{2}{ }^{-*}$ production in platelets in response to the different treatments. All data are presented as means \pm standard deviations. Different letters refer to significant differences, while the same letters do not indicate significant differences among groups.

Comparison of $\mathrm{NO}$ and $\mathrm{O}_{2}^{-*}$ contents in each group of platelets

Decreased NO contents and increased $\mathrm{O}_{2}{ }^{-}$contents were detected in the ox-LDL group and Rapamycin group compared to the Control group and ox-LDL group, respectively, as shown in Fig. 4 (all $P<0.05$ ). Compared with the ox-LDL group, the NAC group, 3-MA group and NAC + 3-MA group exhibited a substantial increase in the NO content and a noticeable

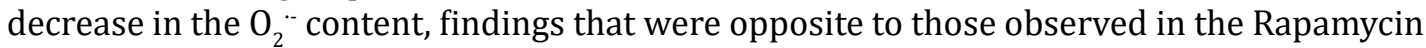
group (all $P<0.05$ ). Additionally, the NO content was higher but the $\mathrm{O}_{2}{ }^{--}$content was lower in both the Rapamycin + 3-MA group and NAC + Rapamycin group than in Rapamycin group (both $P<0.05$ ).

Observation of autophagosomes and expression of the autophagy-related protein LC3

As shown in Fig. 5A, the Control group exhibited the normal, long, strip-shaped mitochondria with a clean ridge structure, but no autophagosomes, while autophagosomes were present in the ox-LDL group under the transmission electron microscope. Furthermore, a large number of autophagosomes with distinct double membranes or myelin figures was present and accumulated in the Rapamycin group. Compared with the ox-LDL group, the number of autophagosomes was significantly decreased in the NAC group, 3-MA group and $\mathrm{NAC}+3$-MA group (both $P<0.05$ ). Immunofluorescence staining was used to observe the 


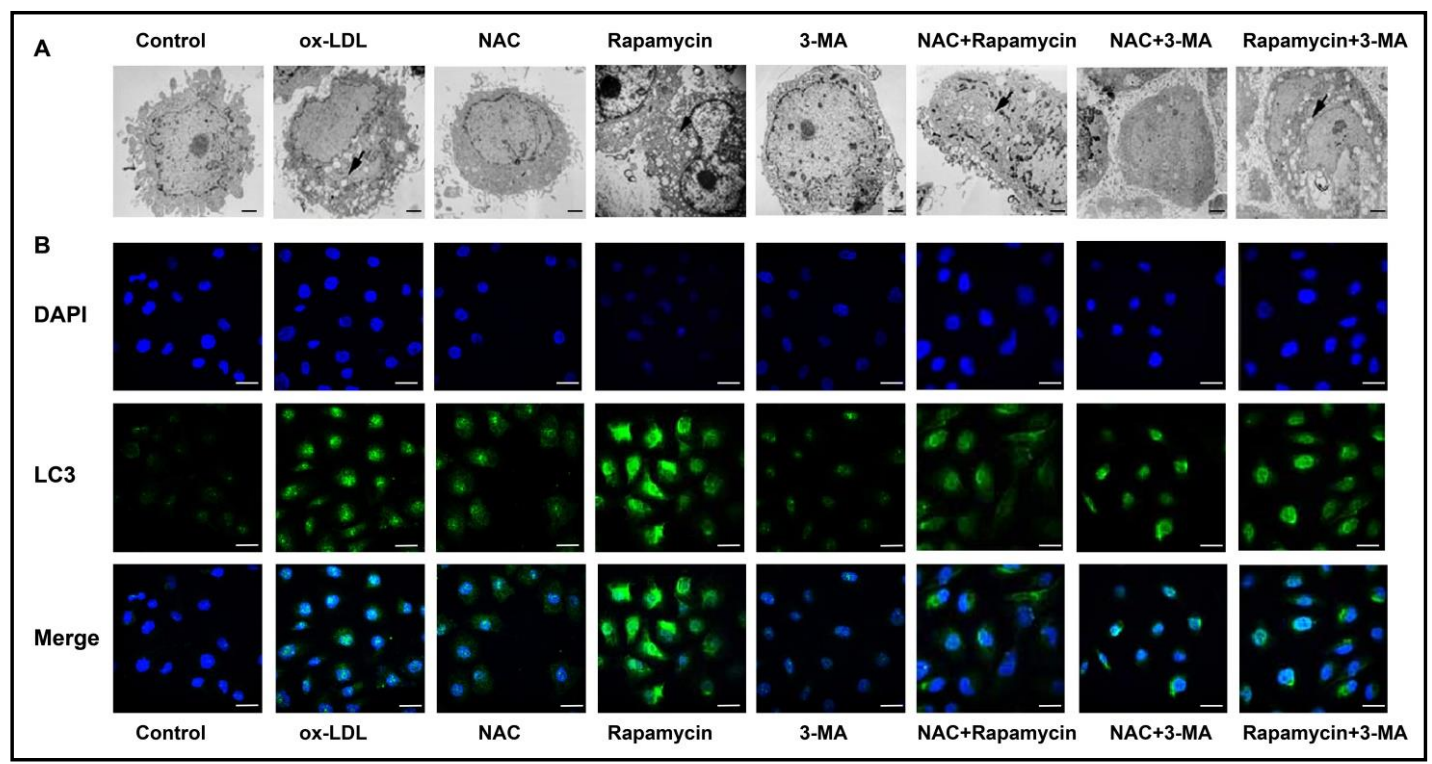

Fig. 5. Observation of autophagosomes and distribution of the autophagy-related protein LC3 . Notes: A, Morphological alterations in platelets occurring in response to the various treatments were detected by transmission electron microscopy (the black arrow indicates autophagosomes, scale bar: $2 \mu \mathrm{m}$ ). B, Platelets treated with the different compounds were stained with an anti-LC3 antibody and DAPI and observed under a fluorescence microscope (scale bar: $20 \mu \mathrm{m}$ ).

Fig. 6. Western blot analysis of the levels of autophagy-related proteins in each group of platelets. Notes: Levels of the LC3-I, LC3-II, Beclin1, and p62 proteins were analyzed by Western blotting, and quantification of the LC3 II/LC3-I ratio, Beclin 1, and p62 levels are shown. All data are presented as means \pm standard deviations. Different letters refer to significant differences, while the same letters do not indicate significant differences among groups.

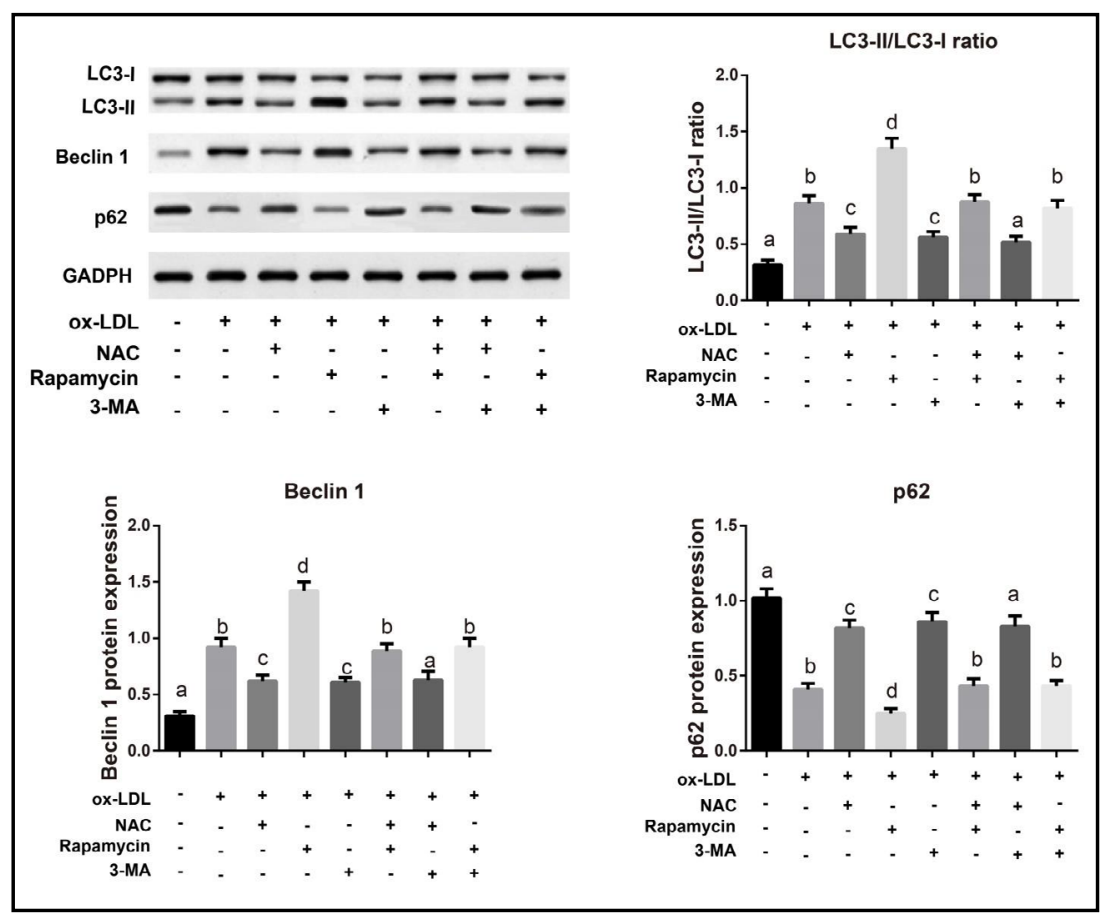

distribution of LC3. No LC3 green fluorescence was detected in the Control group, while the ox-LDL group displayed an increase in LC3 expression, which was distributed around the nucleus in the cytoplasm and formed various scattered green fluorescent dots. However, LC3 expression was significantly increased in the Rapamycin group, but was reduced in the NAC group, 3-MA group and NAC + 3-MA group compared with the ox-LDL group (all $P<0.05$ ). In contrast to the Rapamycin group, LC3 expression was dramatically decreased in the NAC + Rapamycin group and Rapamycin +3 -MA group (both $P<0.05$, Fig. 5B). 


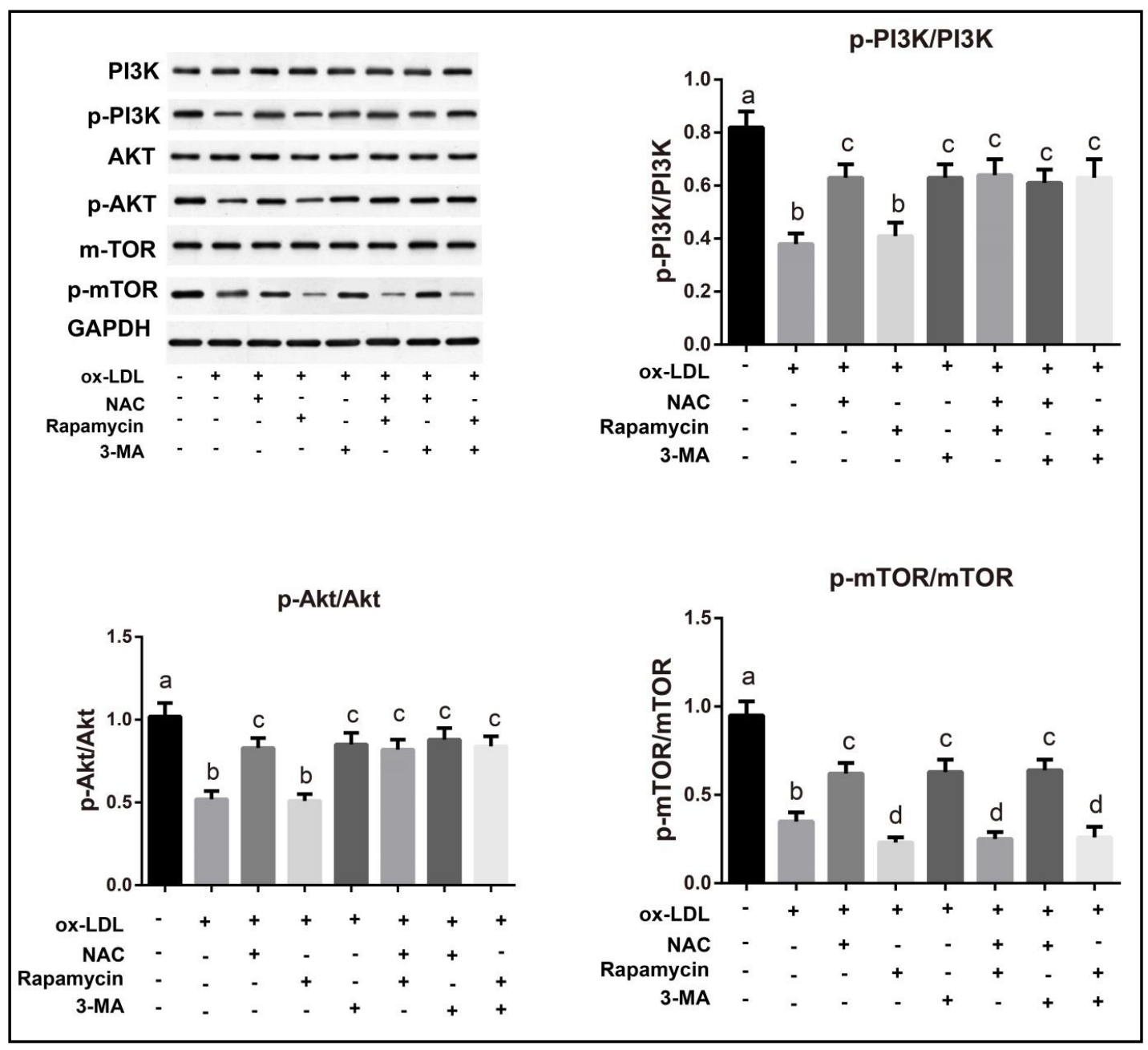

Fig. 7. Western blot analysis of the levels of PI3K/AKT/mTOR pathway-related proteins in each group of platelets. Notes: Levels of the PI3K, p-PI3K, AKT, p-AKT, mTOR, and p-mTOR proteins in the different groups were analyzed by Western blotting, and the quantification of the PI3K/p-PI3K ratio, p-AKT/AKT ratio and $\mathrm{p}$-mTOR/mTOR ratio is shown. All data are presented as means \pm standard deviations. Different letters refer to significant differences, while the same letters do not indicate significant differences among groups.

Levels of autophagy-related proteins in each group of platelets

A significant up-regulation of LC3II/LC3I and Beclin1 and a substantial down-regulation of p62 were observed in the ox-LDL group and Rapamycin group compared with the Control group and ox-LDL group, respectively (all $P<0.05$ ). Additionally, LC3II/LC3I and Beclin1 expression were down-regulated in the NAC group, 3-MA group and NAC + 3-MA group, but p62 expression was up-regulated. These changes differed from the changes observed in the Rapamycin group compared with the ox-LDL group (all $P<0.05$ ). Moreover, lower levels of LC3II/LC3I and Beclin1, and higher levels of p62 were observed in the NAC + rapamycin group and Rapamycin +3 -MA group than Rapamycin group (all $P<0.05$, Fig. 6).

\section{Levels of PI3K/AKT/mTOR pathway-related proteins in each group of platelets}

Western blotting was used to detect the levels of PI3K/AKT/mTOR pathway-related proteins in each group. As shown in Fig. 7, evident decreases in the levels of p-PI3K/PI3K, $\mathrm{p}$-AKT/AKT and p-mTOR/mTOR were observed in the ox-LDL group and Rapamycin group compared with the Control group (all $P<0.05$ ). Additionally, much higher levels of p-PI3K/ PI3K and p-AKT/AKT were observed in the NAC, 3-MA, NAC + 3-MA, NAC + Rapamycin and 
Rapamycin + 3-MA groups than in the ox-LDL group (all $P<0.05$ ). In addition, in contrast to the ox-LDL group, p-mTOR/mTOR levels were significantly increased in the NAC, 3-MA, and $\mathrm{NAC}+3$-MA groups, but were dramatically decreased in the Rapamycin, NAC + Rapamycin, and rapamycin +3 -MA groups (all $P<0.05$ ).

\section{Discussion}

To our knowledge, platelet activation and aggregation are recognized as complex and key processes involved in thrombus formation after the rupture of an atherosclerotic plaque. Meanwhile, ox-LDL is not only important for cardiovascular diseases but is also an independent inducer of platelet activation. Specifically, ox-LDL increases platelet activity and promotes platelet adhesion, aggregation and release by directly interacting with flowing platelets in circulating blood [29,30]. Notably, alterations in platelet membrane glycoproteins are specific molecular markers of activated platelets. One of these markers is CD62p (also called P-selectin), a glycoprotein that was initially identified on activated platelet membranes by Blann et al. [31], and was only selectively distributed on $\alpha$ particles in resting platelets, but was not expressed or was expressed at persistently low levels on the surface of normal platelets [32]. However, CD62p was redistributed in activated platelets, thereby mediating the adhesion and interaction of platelets with granulocytes and endothelial cells to facilitate platelet-monocyte aggregation and accelerate intravascular thrombosis [33, 34]. In the present study, ox-LDL induced platelet activation in vitro, significantly increased platelet aggregation and adhesion, and dramatically increased the percentage of platelets expressing the activation marker CD62p, suggesting that ox-LDL promotes platelet adhesion, aggregation, release and the deformation of particles, contributing to the occurrence of coronary heart disease and thrombus [35]. Additionally, ROS and $\mathrm{O}_{2}^{-}$levels were noticeably increased but the NO content was substantially decreased in ox-LDL-treated platelets. A close relation between ROS and platelets has been reported, and ROS (including $\mathrm{O}_{2}^{-}, \mathrm{OH}^{-}$or $\mathrm{H}_{2} \mathrm{O}_{2}$ ) function as second messengers and are produced by resting or thrombin-activated platelets [36]. Once platelets are stimulated with certain agonists, the nicotinamide adenine dinucleotide (phosphate) (NAD(P)H) oxidases are specifically activated [37], further generating ROS and $\mathrm{O}_{2}^{-}$[38]. In addition, the enzyme nitric oxide synthase (NOS) determines NO formation through the oxidation of L-arginine in platelets [39]. Ox-LDL-mediated eNOS uncoupling inhibits NO generation [40]. Moreover, the uncoupling of eNOS leads to higher $\mathrm{O}_{2}^{-}$and lower NO production under oxidative stress conditions [41], indicating that an oxLDL treatment may decrease NO production and increase $\mathrm{O}_{2}-$ by inhibiting eNOS activation. More importantly, Rapamycin-treated platelets exhibited higher $\mathrm{O}_{2}^{-}$levels and lower NO production than cells treated with ox-LDL alone in our study. Notably, rapamycin-mediated decreases in the levels of the eNOS mRNA and protein have been documented in several studies. For example, the Rapamycin-induced reduction in eNOS production in endothelial progenitor cells occurred in a concentration-/time-dependent manner in a previous study by Chen TG et al. [42]. According to David C. Reineke et al. [43], treatment of endothelial cells with Rapamycin significantly reduced eNOS release and activity in a concentrationdependent manner by inhibiting the PKB/Akt pathway, which activate mTOR, ultimately leading to the decreased production of NO $[44,45]$ and further suggesting that rapamycin may decrease NO production and stimulate $\mathrm{O}_{2}$ - production owing to reduce eNOS levels.

Autophagosome formation, increased levels of LC3II/LC3I and Beclin1, and decreased levels of p62 were also simultaneously observed in ox-LDL-treated platelets, implying that activated platelets underwent autophagy, consistent with the results from a previous study [15]. Generally, ROS production may induce autophagy to overcome oxidative stress [46, 47] by specifically inactivating Atg4 to cause the accumulation of LC3II and increase the formation of autophagosomes [48]. On the other hand, ROS contribute to the ubiquitination of the degraded substances, and p62 interacts with LC3 (a member of a novel ubiquitin-like protein family) to promote its degradation by the autophagosome-lysosome system [49].

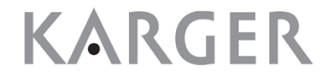


Moreover, ox-LDL induces autophagy, as evidenced by increased LC3 and Beclin-1 expression [50]. More importantly, ROS induce autophagy by modulating the activity of related signaling pathways. For example, in the study by Mi et al., Momordin Ic induced autophagy by inhibiting the ROS-mediated activation of the PI3K/AKT pathway, and apoptosis was also suppressed by the autophagy inhibitor 3-MA [51]. Additionally, ROS overproduction led to the activation of autophagy by suppressing the PI3K/AKT/mTOR signaling pathway, which plays a critical role in regulating autophagy [52]. Consistent with these results, our findings revealed the restricted activity of PI3K/AKT/mTOR in activated platelets induced by ox-LDL, indicating that platelet activation increased platelet oxidative stress and ROS levels, thereby promoting platelet autophagy possibly via the inhibition of PI3K/AKT/mTOR signaling.

We applied the ROS scavenger NAC, autophagy inhibitor 3-MA and/or mTOR inhibitor Rapamycin to activated platelets to clarify the regulatory mechanism underlying platelet autophagy. Both NAC and 3-MA significantly inhibited activated platelet aggregation and adhesion, along with decreasing ROS levels and the expression of LC3II/LC3I and Beclin1, elevating p62 levels, and activating the PI3K/AKT/mTOR signaling pathway. In contrast, Rapamycin reversed these processes to inhibit the phosphorylation of mTOR and facilitate the activation and autophagy of platelets. Traditionally, PI3K, a class I PI3K in the PI3K/AKT/ mTOR signaling pathway, activates the serine/threonine kinase AKT, and then phosphorylates mTOR via a series of regulatory steps, ultimately inhibiting autophagy [53]. Akt, a member of the family of serine/threonine protein kinases, is activated in a PI3K-dependent manner in response to various stimuli [54], while the phosphorylated class I PI3K enzymes and protein kinase B (Akt/PKB) also activate mTOR [55]. As one of the downstream signaling targets of PI3K/Akt, mTOR was suggested to regulate the signaling proteins responsible for protein synthesis, such as ribosomal p70S6 kinase [56],indicating that during platelet activation, Rapamycin induces the autophagy reaction that has been repressed by class I PI3K by inhibiting the phosphorylation of mTOR. In contrast, class III PI3K (Vps34) interacts with Beclin1 and Vps15 to form class III PI3KC, which initiates autophagy during the formation of the pre-autophagy bilayer membrane in the early stage of autophagy [57]. As discussed above, starvation or the mTOR inhibitor Rapamycin trigger platelet autophagy to induce platelet aggregation and adhesion [16]. Furthermore, a subsequent study confirmed that 3-MA suppresses autophagy by inhibiting class III PI3K [58]. The inhibitor of class III PI3K 3-MA blocks the plasma membrane translocation of important cytoplasmic subunits of NOX2 NADPH oxidase, further affecting the assembly and activation of NOX2 complex on the platelet membrane and subsequently reducing ROS production, ultimately affecting platelet activation and thrombosis [59]. Furthermore, the NOX2 NADPH was identified as one of the key sources of ROS, and a recent study has revealed a novel role for the NOX2 NADPH oxidase in the activation of autophagy [60]. As shown in the present study, platelets treated with 3-MA exhibited lower ROS and autophagy levels, suggesting that 3-MA blocks autophagy by inhibiting class III PI3K activity, which may block NOX2-derived ROS production. More importantly, this study confirmed the hypothesis that Rapamycin-induced autophagy is blocked by the ROS scavenger NAC and autophagy inhibitor 3-MA. In the study by Roy et al., zinc oxide nanoparticles induced ROS formation in macrophages to inhibit the PI3K/AKT/ mTOR signaling pathway and subsequently promote cell autophagy and apoptosis, whereas NAC blocked ROS production, cell autophagy and apoptosis [61], further indicating that inhibition of the activity of the PI3K/AKT/mTOR pathway is partially regulated by ROS and subsequently promotes autophagy in activated platelets.

\section{Conclusion}

Taken together, as illustrated schematically in Fig. 8, the present study has revealed that ox-LDL-induced oxidative stress triggered platelet activation and increased ROS production, thereby suppressing the downstream activity of the PI3K/AKT/mTOR signaling pathway, promoting platelet autophagy, and consequently exacerbating platelet aggregation and

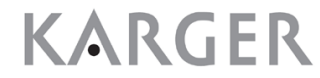




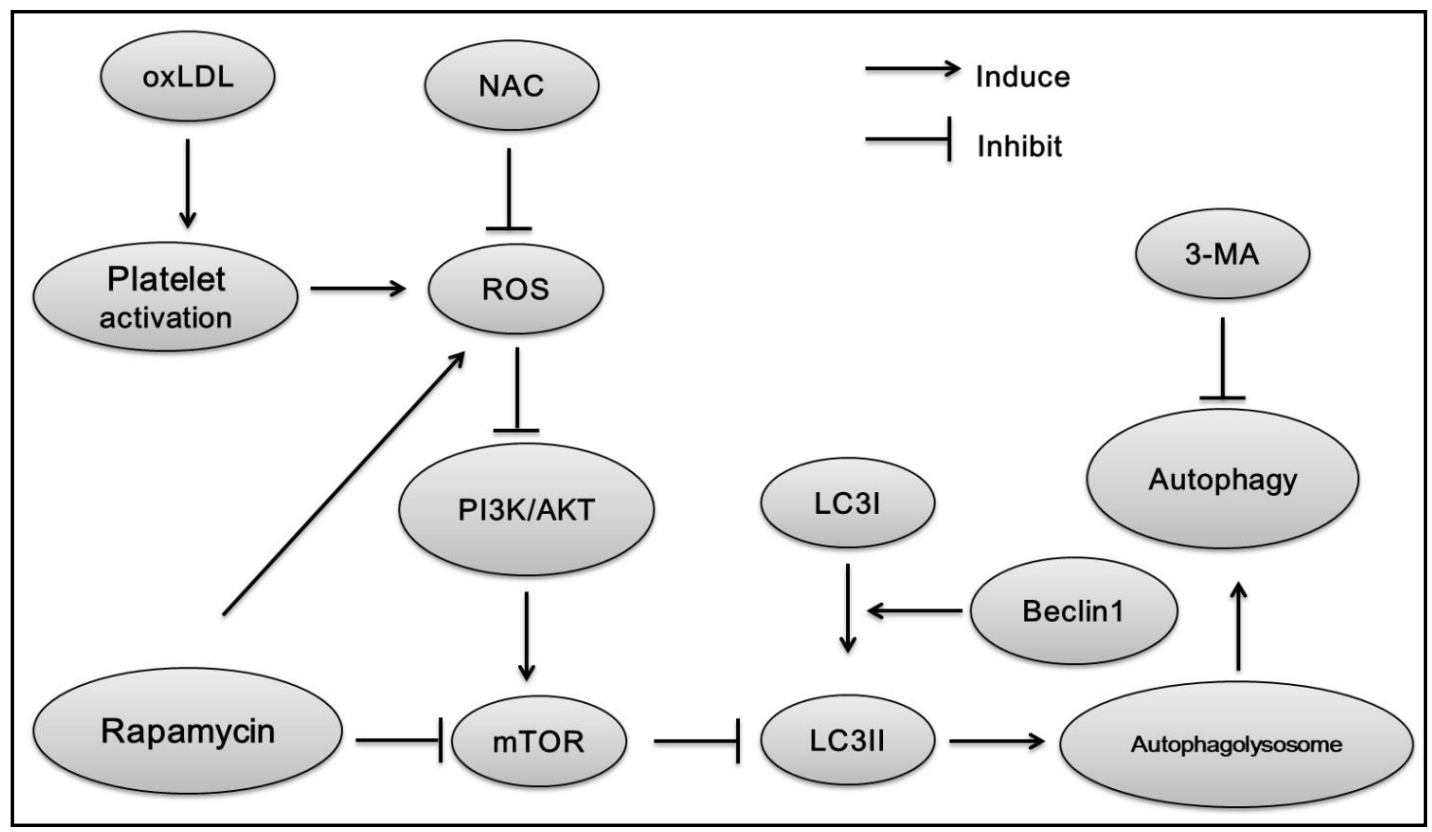

Fig. 8. Schematic illustrating the proposed mechanism by which ROS induce autophagy to promote ox-LDLinduced platelet activation by inhibiting the PI3K/AKT/mTOR pathway.

adhesion. However, strategies that repress ROS activity may influence the platelet NO and $\mathrm{O}_{2}-$ contents to reduce autophagy through the activation of the PI3K/AKT/mTOR pathway, thus blocking platelet activation to a certain extent and ultimately achieving the beneficial effects of inhibiting platelet aggregation.

\section{Acknowledgements}

We would like to express our sincere appreciation to the reviewers for their constructive comments on this work.

\section{Disclosure Statement}

The authors have no conflicts of interest.

\section{References}

$>1$ Steinberg D: Hypercholesterolemia and inflammation in atherogenesis: two sides of the same coin. Mol Nutr Food Res 2005;49:995-998.

2 Writing Group M, Lloyd-Jones D, Adams RJ, Brown TM, Carnethon M, Dai S, De Simone G, Ferguson TB, Ford E, Furie K, Gillespie C, Go A, Greenlund K, Haase N, Hailpern S, Ho PM, Howard V, Kissela B, Kittner S, Lackland D et al.: Heart disease and stroke statistics--2010 update: a report from the American Heart Association. Circulation 2010;121:e46-e215.

- 3 Badrnya S, Assinger A, Volf I: Native high density lipoproteins (HDL) interfere with platelet activation induced by oxidized low density lipoproteins (OxLDL). Int J Mol Sci 2013;14:10107-10121.

4 Wierzbicki AS, Viljoen A, Hardman TC, Mikhailidis DP: New therapies to reduce low-density lipoprotein cholesterol. Curr Opin Cardiol 2013;28:452-457.

5 Shishehbor MH, Hazen SL: Inflammatory and oxidative markers in atherosclerosis: relationship to outcome. Curr Atheroscler Rep 2004;6:243-250. 


\section{Cellular Physiology Cell Physiol Biochem 2018;50:1779-1793

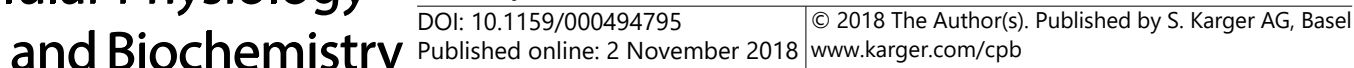

Wang et al.: ROS in Ox-LDL-Induced Platelet Activation

-6 Magwenzi S, Woodward C, Wraith KS, Aburima A, Raslan Z, Jones H, McNeil C, Wheatcroft S, Yuldasheva N, Febbriao M, Kearney M, Naseem KM: Oxidized LDL activates blood platelets through CD36/NOX2-mediated inhibition of the cGMP/protein kinase G signaling cascade. Blood 2015;125:2693-2703.

7 Carnevale R, Bartimoccia S, Nocella C, Di Santo S, Loffredo L, Illuminati G, Lombardi E, Boz V, Del Ben M, De Marco L, Pignatelli P, Violi F: LDL oxidation by platelets propagates platelet activation via an oxidative stress-mediated mechanism. Atherosclerosis 2014;237:108-116.

-8 Peng N, Meng N, Wang S, Zhao F, Zhao J, Su L, Zhang S, Zhang Y, Zhao B, Miao J: An activator of mTOR inhibits oxLDL-induced autophagy and apoptosis in vascular endothelial cells and restricts atherosclerosis in apolipoprotein E(-)/(-) mice. Sci Rep 2014;4:5519.

-9 Stellos K, Sauter R, Fahrleitner M, Grimm J, Stakos D, Emschermann F, Panagiota V, Gnerlich S, Perk A, Schonberger T, Bigalke B, Langer HF, Gawaz M: Binding of oxidized low-density lipoprotein on circulating platelets is increased in patients with acute coronary syndromes and induces platelet adhesion to vascular wall in vivo--brief report. Arterioscler Thromb Vasc Biol 2012;32:2017-2020.

10 Korporaal SJ, Gorter G, van Rijn HJ, Akkerman JW: Effect of oxidation on the platelet-activating properties of low-density lipoprotein. Arterioscler Thromb Vasc Biol 2005;25:867-872.

11 Lievens D, von Hundelshausen P: Platelets in atherosclerosis. Thromb Haemost 2011;106:827-838.

12 Todde V, Veenhuis M, van der Klei IJ: Autophagy: principles and significance in health and disease. Biochim Biophys Acta 2009;1792:3-13.

13 Lin MG, Zhong Q: Interaction between small GTPase Rab7 and PI3KC3 links autophagy and endocytosis: A new Rab7 effector protein sheds light on membrane trafficking pathways. Small GTPases 2011;2:85-88.

14 Mizushima N, Levine B, Cuervo AM, Klionsky DJ: Autophagy fights disease through cellular self-digestion. Nature 2008;451:1069-1075.

$>15$ Ouseph MM, Huang Y, Banerjee M, Joshi S, MacDonald L, Zhong Y, Liu H, Li X, Xiang B, Zhang G, Komatsu M, Yue Z, Li Z, Storrie B, Whiteheart SW, Wang QJ: Autophagy is induced upon platelet activation and is essential for hemostasis and thrombosis. Blood 2015;126:1224-1233.

-16 Feng W, Chang C, Luo D, Su H, Yu S, Hua W, Chen Z, Hu H, Liu W: Dissection of autophagy in human platelets. Autophagy 2014;10:642-651.

17 Hou X, Hu Z, Xu H, Xu J, Zhang S, Zhong Y, He X, Wang N: Advanced glycation endproducts trigger autophagy in cadiomyocyte via RAGE/PI3K/AKT/mTOR pathway. Cardiovasc Diabetol 2014;13:78.

18 Heras-Sandoval D, Perez-Rojas JM, Hernandez-Damian J, Pedraza-Chaverri J: The role of PI3K/AKT/mTOR pathway in the modulation of autophagy and the clearance of protein aggregates in neurodegeneration. Cell Signal 2014;26:2694-2701.

19 Kumar S, Guru SK, Pathania AS, Manda S, Kumar A, Bharate SB, Vishwakarma RA, Malik F, Bhushan S: Fascaplysin induces caspase mediated crosstalk between apoptosis and autophagy through the inhibition of PI3K/AKT/mTOR signaling cascade in human leukemia HL-60 cells. J Cell Biochem 2015;116:985-997.

-20 Jiang Y, Kou J, Han X, Li X, Zhong Z, Liu Z, Zheng Y, Tian Y, Yang L: ROS-Dependent Activation of Autophagy through the PI3K/Akt/mTOR Pathway Is Induced by Hydroxysafflor Yellow A-Sonodynamic Therapy in THP-1 Macrophages. Oxid Med Cell Longev 2017;2017:8519169.

-21 Han XB, Li HX, Jiang YQ, Wang H, Li XS, Kou JY, Zheng YH, Liu ZN, Li H, Li J, Dou D, Wang Y, Tian Y, Yang LM: Upconversion nanoparticle-mediated photodynamic therapy induces autophagy and cholesterol efflux of macrophage-derived foam cells via ROS generation. Cell Death Dis 2017;8:e2864.

22 Gibson KR, Winterburn TJ, Barrett F, Sharma S, MacRury SM, Megson IL: Therapeutic potential of $\mathrm{N}$-acetylcysteine as an antiplatelet agent in patients with type-2 diabetes. Cardiovasc Diabetol 2011;10:43.

23 Jiang P, Lan Y, Luo J, Ren YL, Liu DG, Pang JX, Liu J, Li J, Wang C, Cai JP: Rapamycin promoted thrombosis and platelet adhesion to endothelial cells by inducing membrane remodeling. BMC Cell Biol 2014;15:7.

-24 Aslan JE, Tormoen GW, Loren CP, Pang J, McCarty OJ: S6K1 and mTOR regulate Rac1-driven platelet activation and aggregation. Blood 2011;118:3129-3136.

25 [The Helsinki Declaration of the World Medical Association (WMA). Ethical principles of medical research involving human subjects]. Pol Merkur Lekarski 2014;36:298-301.

-26 Nergiz-Unal R, Lamers MM, Van Kruchten R, Luiken JJ, Cosemans JM, Glatz JF, Kuijpers MJ, Heemskerk JW: Signaling role of CD36 in platelet activation and thrombus formation on immobilized thrombospondin or oxidized low-density lipoprotein. J Thromb Haemost 2011;9:1835-1846.

27 Chen K, Febbraio M, Li W, Silverstein RL: A specific CD36-dependent signaling pathway is required for platelet activation by oxidized low-density lipoprotein. Circ Res 2008;102:1512-1519. 


\section{Cellular Physiology Cell Physiol Biochem 2018;50:1779-1793 and Biochemistry DOI: 10.1159/000494795 2018 (0)18 The Author(s). Published by S. Karger AG, Basel

Wang et al.: ROS in Ox-LDL-Induced Platelet Activation

28 Paul M, Hemshekhar M, Thushara RM, Sundaram MS, NaveenKumar SK, Naveen S, Devaraja S, Somyajit K, West R, Basappa, Nayaka SC, Zakai UI, Nagaraju G, Rangappa KS, Kemparaju K, Girish KS: Methotrexate Promotes Platelet Apoptosis via JNK-Mediated Mitochondrial Damage: Alleviation by N-Acetylcysteine and N-Acetylcysteine Amide. PLoS One 2015;10:e0127558.

29 Podrez EA, Byzova TV, Febbraio M, Salomon RG, Ma Y, Valiyaveettil M, Poliakov E, Sun M, Finton PJ, Curtis BR, Chen J, Zhang R, Silverstein RL, Hazen SL: Platelet CD36 links hyperlipidemia, oxidant stress and a prothrombotic phenotype. Nat Med 2007;13:1086-1095.

-30 Collot-Teixeira S, De Lorenzo F, McGregor JL: Scavenger receptor A and CD36 are implicated in mediating platelet activation induced by oxidized low- density lipoproteins. Arterioscler Thromb Vasc Biol 2007;27:2491-2492.

-31 Blann AD, Lip GY: Hypothesis: is soluble P-selectin a new marker of platelet activation? Atherosclerosis 1997;128:135-138.

-32 Karakantza M, Maniatis A, Metallinos CI, Papapetropoulos T, Paschalis C: In vivo platelet activation in ischemic stroke patients. Stroke 2003;34:e174-175; author reply e174-175.

-33 Haseruck N, Erl W, Pandey D, Tigyi G, Ohlmann P, Ravanat C, Gachet C, Siess W: The plaque lipid lysophosphatidic acid stimulates platelet activation and platelet-monocyte aggregate formation in whole blood: involvement of P2Y1 and P2Y12 receptors. Blood 2004;103:2585-2592.

34 Cerletti C, de Gaetano G, Lorenzet R: Platelet - leukocyte interactions: multiple links between inflammation, blood coagulation and vascular risk. Mediterr J Hematol Infect Dis 2010;2:e2010023.

-35 Langer HF, Bigalke B, Seizer P, Stellos K, Fateh-Moghadam S, Gawaz M: Interaction of platelets and inflammatory endothelium in the development and progression of coronary artery disease. Semin Thromb Hemost 2010;36:131-138.

-36 Freedman JE: Oxidative stress and platelets. Arterioscler Thromb Vasc Biol 2008;28:s11-16.

-37 Jackson SP, Nesbitt WS, Westein E: Dynamics of platelet thrombus formation. J Thromb Haemost 2009;7 Suppl 1:17-20.

-38 Stef G, Csiszar A, Ziangmin Z, Ferdinandy P, Ungvari Z, Veress G: Inhibition of NAD(P)H oxidase attenuates aggregation of platelets from high-risk cardiac patients with aspirin resistance. Pharmacol Rep 2007;59:428-436.

-39 Gresele P, Pignatelli P, Guglielmini G, Carnevale R, Mezzasoma AM, Ghiselli A, Momi S, Violi F: Resveratrol, at concentrations attainable with moderate wine consumption, stimulates human platelet nitric oxide production. J Nutr 2008;138:1602-1608.

-40 Ryoo S, Bhunia A, Chang F, Shoukas A, Berkowitz DE, Romer LH: OxLDL-dependent activation of arginase II is dependent on the LOX-1 receptor and downstream RhoA signaling. Atherosclerosis 2011;214:279-287.

41 Kar S, Bhandar B, Kavdia M: Impact of SOD in eNOS uncoupling: a two-edged sword between hydrogen peroxide and peroxynitrite. Free Radic Res 2012;46:1496-1513.

42 Chen TG, Chen JZ, Wang XX: Effects of rapamycin on number activity and eNOS of endothelial progenitor cells from peripheral blood. Cell Prolif 2006;39:117-125.

43 Reineke DC, Muller-Schweinitzer E, Winkler B, Kunz D, Konerding MA, Grussenmeyer T, Carrel TP, Eckstein FS, Grapow MT: Rapamycin impairs endothelial cell function in human internal thoracic arteries. Eur J Med Res 2015;20:59.

44 Dormond 0, Madsen JC, Briscoe DM: The effects of mTOR-Akt interactions on anti-apoptotic signaling in vascular endothelial cells. J Biol Chem 2007;282:23679-23686.

45 Contreras AG, Dormond O, Edelbauer M, Calzadilla K, Hoerning A, Pal S, Briscoe DM: mTOR-understanding the clinical effects. Transplant Proc 2008;40:S9-S12.

46 Filomeni G, De Zio D, Cecconi F: Oxidative stress and autophagy: the clash between damage and metabolic needs. Cell Death Differ 2015;22:377-388.

47 Scherz-Shouval R, Shvets E, Elazar Z: Oxidation as a post-translational modification that regulates autophagy. Autophagy 2007;3:371-373.

48 Scherz-Shouval R, Shvets E, Fass E, Shorer H, Gil L, Elazar Z: Reactive oxygen species are essential for autophagy and specifically regulate the activity of Atg4. EMBO J 2007;26:1749-1760.

49 Lee J, Giordano S, Zhang J: Autophagy, mitochondria and oxidative stress: cross-talk and redox signalling. Biochem J 2012;441:523-540.

50 Muller C, Salvayre R, Negre-Salvayre A, Vindis C: HDLs inhibit endoplasmic reticulum stress and autophagic response induced by oxidized LDLs. Cell Death Differ 2011;18:817-828. 


\section{Cellular Physiology Cell Physiol Biochem 2018;50:1779-1793 and Biochemistry $\begin{aligned} & \text { DOl: 10.1159/000494795 } \\ & \text { Published }\end{aligned}$ \\ Wang et al.: ROS in Ox-LDL-Induced Platelet Activation}

51 Mi Y, Xiao C, Du Q, Wu W, Qi G, Liu X: Momordin Ic couples apoptosis with autophagy in human hepatoblastoma cancer cells by reactive oxygen species (ROS)-mediated PI3K/Akt and MAPK signaling pathways. Free Radic Biol Med 2016;90:230-242.

52 Portal-Nunez S, Esbrit P, Alcaraz MJ, Largo R: Oxidative stress, autophagy, epigenetic changes and regulation by miRNAs as potential therapeutic targets in osteoarthritis. Biochem Pharmacol 2016;108:110.

-53 Rodon J, Dienstmann R, Serra V, Tabernero J: Development of PI3K inhibitors: lessons learned from early clinical trials. Nat Rev Clin Oncol 2013;10:143-153.

-54 Testa JR, Bellacosa A: AKT plays a central role in tumorigenesis. Proc Natl Acad Sci U S A 2001;98:1098310985.

-55 Zhai C, Cheng J, Mujahid H, Wang H, Kong J, Yin Y, Li J, Zhang Y, Ji X, Chen W: Selective inhibition of PI3K/ Akt/mTOR signaling pathway regulates autophagy of macrophage and vulnerability of atherosclerotic plaque. PLoS One 2014;9:e90563.

56 Kesari S, Ramakrishna N, Sauvageot C, Stiles CD, Wen PY: Targeted molecular therapy of malignant gliomas. Curr Neurol Neurosci Rep 2005;5:186-197.

57 Itakura E, Mizushima N: Characterization of autophagosome formation site by a hierarchical analysis of mammalian Atg proteins. Autophagy 2010;6:764-776.

58 Wu YT, Tan HL, Shui G, Bauvy C, Huang Q Wenk MR, Ong CN, Codogno P, Shen HM: Dual role of 3-methyladenine in modulation of autophagy via different temporal patterns of inhibition on class I and III phosphoinositide 3-kinase. J Biol Chem 2010;285:10850-10861.

59 Liu Y, Hu M, Luo D, Yue M, Wang S, Chen X, Zhou Y, Wang Y, Cai Y, Hu X, Ke Y, Yang Z, Hu H: Class III PI3K Positively Regulates Platelet Activation and Thrombosis via PI(3)P-Directed Function of NADPH Oxidase. Arterioscler Thromb Vasc Biol 2017;37:2075-2086.

-60 Lam GY, Huang J, Brumell JH: The many roles of NOX2 NADPH oxidase-derived ROS in immunity. Semin Immunopathol 2010;32:415-430.

61 Roy R, Singh SK, Chauhan LK, Das M, Tripathi A, Dwivedi PD: Zinc oxide nanoparticles induce apoptosis by enhancement of autophagy via PI3K/Akt/mTOR inhibition. Toxicol Lett 2014;227:29-40. 